\title{
Catastrophic Failures Propagate Field of Fracture Mechanics
}

The development of fracture mechanics dates back five centuries to Leonardo da Vinci, who carried out experiments on the fracture of iron wire. He demonstrated that the strength of a piece of wire varies inversely with its length; long wires are weaker, on average, while short wires are stronger. This behavior, better known today as the "size effect," supported the theory that wire fracture is initiated by rare flaws. A longer piece of wire is more likely to contain a larger flaw that causes fracture at a lower critical stress.

More systematic investigations of fracture phenomena came only in the nineteenth and twentieth centuries. Not surprisingly, much of the development of modern fracture mechanics was motivated by catastrophic fracture-related accidents. In the 1800 s more than two hundred steamboats suffered boiler explosions, including the Soltana, a Civil War steamboat whose explosion resulted in the deaths of over a thousand Union soldiers. In 1919, a Boston molasses tank ruptured, and a two-million-gallon flood of the viscous, sticky fluid drowned twelve people.

In the $1940 \mathrm{~s}, 2,500$ Liberty ships were built to aid Britain in World War II; of these, about 700 sustained serious cracks and 145 broke completely in half. In the 1950s two Comet jet aircraft experienced catastrophic failures, breaking apart at high altitude; losing the Comet meant that Britain lost its early competitive position in commercial jet aviation.

Each of these disasters pointed out unforeseen errors in structural design and engineering, and resulted in significant loss of life and property. In each case an engineering structure was designed and built with insufficient knowledge of materials properties and with incomplete understanding of the consequences of design choices. The problem was clear: Many of the "high strength" materials in use could fracture at relatively low applied stress. To prevent future accidents, scientists and engineers undertook serious investigation of the mechanisms by which cracks grew in different materials under various conditions of stress, temperature, and environment.

Modern scientific study of fracture began in 1920, when A. A. Griffith developed an energy criterion for crack growth, concluding that for a given stress, there is a critical size above which a crack will propagate in an unstable fashion. For a crack of critical size, crack propagation releases just enough strain energy to cancel out the surface energy cost. This work drew on an earlier result of C.E. Ingliss who performed a stress analysis for an elastic medium containing an elliptical hole. Griffith's model applied well to glasses, and could predict the fracture stress of a glass sample as a function of the size of its largest flaw. However, because Griffith's model relied on the assumption of linear elasticity, it did not properly describe the fracture behavior of ductile materials, such as many metals, where plastic deformation plays an important role.

In the late 1940s, G.R. Irwin of the U.S. Naval Research Laboratory and E. Orowan of MIT both independently reformulated Griffith's theory to include the effects of plastic deformation. Later, Irwin introduced the concept of the energy release rate, and developed the idea of a stress intensity factor characterizing the stress concentration singularity near a crack tip. Irwin's contributions formed the backbone of modern fracture mechanics, and made possible for the first time the practical application of fracture mechanics to engineering design.

In 1956, A.A. Wells successfully applied these ideas to explain the structural failure of the Comet jet aircraft. He showed that stress concentration near the sharp corner of a square window led to initiation of fatigue cracks. Under repeated stress cycles, these cracks grew to a critical size and caused the fuselage to crack apart. In 1957, D.H. Winne and B.M. Wundt of General Electric Co. made another important application of fracture mechanics ideas in an analysis of the failure of large rotors in steam turbines.

Similar analysis explained the failure of the Liberty ships. Most of the fractures had initiated at square hatch corners or square cutouts. An obvious solution was to change the design to use only rounded, reinforced corners (which may be evident in the shape of the windows on a commercial jet). But that was not the only problem. Some of the steel used in construction was brittle at ocean temperatures, and to make matters worse, the welded design did not include rivets which could act as crack arresters. Ruptures of steam boilers and the famous Boston molasses tank are harder to explain simply because of the broad range of possible mechanisms.

From these early beginnings, fracture mechanics has grown to become a mature engineering discipline. Stress analysis is a routine but critical step in the design of any structural element, and engineers make use of finite element methods to model stress distributions in proposed designs for everything from aircraft to skyscrapers. The fracture mechanics of metals, ceramics, and polymers have been characterized, both with and without the chemical effects of environmental agents, such as stress corrosion. Laboratory measurements of materials properties such as fracture toughness enable engineers to choose the right material for particular applications. Very strong materials and coatings have been developed that can function under extreme stress and/or temperature conditions.

In spite of these advancements, serious accidents due to materials failure still happen when a new failure scenario arises, such as in the case of an Aloha Airlines plane in 1988. Because it was used for short trips between neighboring islands in Hawaii, the aircraft had performed an unusually large number of take-offs and landings for its age. The resulting pressure cycling is known to cause fatigue cracks emanating from the rivet holes in the aircraft's skin. But the Aloha plane had been designed to resist unstable fracture so that any isolated crack in the structure's skin would arrest at a point where the skin was riveted to the underlying ribs.

Though the plane's designers had tried to plan for every possible circumstance, the aircraft eventually failed in an unforeseen manner, via the link-up of fatigue cracks located at multiple sites along the fuselage. This link-up process in the case of "multiple-site damage" occurs because cracks in close proximity interact, increasing the stress intensity on both and resulting in cooperative growth. When many small cracks joined together to make a large crack, a large section of the Aloha plane's fuselage suddenly ripped away in flight. While the pilot was able to land the 
badly damaged plane, one death resulted from the accident. Since then engineers have focused on advanced techniques to detect multiple-site damage in existing aircraft (through ultrasound and tomographic methods), and on the refinement of multiple-site damage/fracture mechanics models. The accident has also spurred both government and industry scientists to take renewed interest in the problems of maintaining the nation's aging aircraft.

Many basic questions remain in the study of fracture. Why do cracks bifur- cate? When is linear crack propagation stable, and when unstable? Why do some materials fracture in a brittle fashion while others are ductile and cannot sustain a sharp crack? Why are metals embrittled by hydrogen? How does a crack interact with the underlying microstructure of a solid, or with interfaces in a composite or layered material? A U.S. government study estimated that in 1978, fracture cost the U.S. economy $\$ 119$ billion (in 1982 dollars). Further research efforts in the area of fracture mechanics thus have the potential to both prevent needless deaths and injuries and to improve the nation's economic well-being.

Robin L. Blumberg SELINGER

FOR Further READING: T.L. Anderson, Fracture Mechanics: Fundamentals and Applications, Chapter 1 (CRC Press, Boca Raton, 1995); and John M. Barsom, ed., Fracture Mechanics Retrospective: Early Classic Papers (1913-1965) (American Society for Testing and Materials, Philadelphia, 1987).

\section{Chemical Processing of Ceramics Edited by B.I. Lee and E.J.A. Pope (Marcel Dekker, 1994) ISBN: 0-8247-9244-0}

Chemical Processing of Ceramics is an eclectic collection of articles on various aspects of the use of chemistry in preparing materials. The book has six sections: Precursor Chemistry, Powder Synthesis and Characterization, Powder Processing, Sol-Gel Processing, Ceramics via Polymer Chemistry, and Processing of Specialty Ceramics. Each contains a number of chapters devoted to specific subjects. As noted in the foreword by P.E.D. Morgan, the use of chemical routes to materials synthesis is often viewed as costly. Yet, he comments that industrial processes can often overcome such difficulties and notes that the largest volume of silicon nitride is currently produced by the chemical reaction of silicon tetrachloride and ammonia.

The book considers many of the major chemical processing routes to ceramic materials with sufficient detail to give the reader an appreciation for each subject. Rather than a manual for proceeding to utilize the approaches, which no realistic single book could accomplish, it provides a clear assessment of the potential of the techniques. Thus it is an invitation to the reader to begin to assess the alternatives and take seriously the attractive attributes of chemical processing.

One of the major advantages of chemical processing is the atomic level mixing of constituents that allows processing at lower temperatures. This theme recurs in the book, with many of the discussions focused on materials having two or more metal components. For example, in the chapter entitled "Chemical Synthesis of Metal Oxide Powders," C.N.R. Rao emphasizes the preparation of complex, multicomponent oxide powders with con- trolled stoichiometry.

The specificity of the chapters varies widely, ranging from broad coverage such as in "Molecular Design of Transition Metal Alkoxide Precursors" to the very narrow, such as "Metal Alkoxides for Electrooptical Ceramics." The chemical route to powder synthesis, characterization, and processing is fundamental to ceramics as an alternative to the "bruteforce" processing of mechanical mixing and high temperature reaction. Chemical techniques, however, yield greater control over composition, morphology, particle size and distribution, and surface chemistry. Additionally, chemical synthesis allows preparation of nanoscale materials impossible to obtain by most other means.

In the coverage of sol-gel and polymer processing we see the substantial advantages of these routes for specific materials. Through chemical control we may create not only the materials of interest, but may fairly easily tailor their micro- and macrostructure. Thus, we are able to fabricate useful materials ranging from thin films to fiber-reinforced composites.

The final section contains an interesting mix of ceramics prepared via chemical routes for very specific applications. The six chapters cover materials such as leadbased dielectrics, magnetic particles, and ceramic membranes. Examples such as how surface charge affects the packing of nanoscale particles, and thus the permeability of a membrane, are particularly enlightening.

One area of chemical processing that was largely ignored was chemical vapor processes. The technique and its variants is a mainstay of processing for many electronic materials. Alas, however, no book can cover every topic.

Reviewer: T.M. Besmann is a group leader in the Metals and Ceramics Division at Oak
Ridge National Laboratory. His research interests are in the vapor phase processing of ceramic films and composites, and thermochemical analysis and phase equilibria.

\section{Glassy Metals III: Amorphization Techniques, Catalysis, Electronic and lonic Structure \\ Edited by H. Beck and H-J. Güntherodt (Springer-Verlag, 1994) \\ ISBN: 0-387-57440-9}

Glassy Metals III follows the first two volumes in this series, Glassy Metals I and $I I$, which appeared in 1981 and 1983, respectively. The first two volumes appeared at a time when rapid solidification, as the leading technique to prepare metallic glasses, was still a major research topic in materials science. In 1983 three observations of solid-state amorphization, i.e., interdiffusion of thin film diffusion couples, hydrogenation, and mechanical attrition, stimulated research in this area, and much of the new research on amorphous alloys has focused on solid-state amorphization. Chapter 2 in this volume gives an overview of solid-state amorphization which adequately covers the field but provides information already available elsewhere. The same may be said for Chapter 3, which concentrates on the method of mechanical attrition.

The study of amorphous alloys as catalytic materials has also been a major topic of research for about 10 years. The author of this chapter (4) excellently describes the motivation for using metallic glasses in catalysis. A useful table gives the variety of glassy alloys studied and major reaction products which they catalyze. Glassy alloys in the as-quenched state and where they were subjected to various pretreatments (such as reduction in hydrogen, etching, and oxidation) and serve as precursors to catalytically active materials are 